\title{
Vortex Tubes in Turbulent Flows: Identification, Representation, Reconstruction
}

\author{
David C. Banks * \\ Institute for Computer Applications in Science and Engineering \\ Mail Stop 132C, NASA Langley Research Center \\ Hampton, VA 23681 \\ Bart A. Singer ** \\ High Technology Corporation \\ Mail Stop 156, NASA Langley Research Center \\ Hampton, VA 23681
}

\begin{abstract}
In many cases the structure of a fluid flow is well-characterized by its vortices, especially for the purpose of visualization. In this paper we present a new algorithm for identifying vortices in complex flows. The algorithm produces a skeleton line along the center of a vortex by using a two-step predictor-corrector scheme. The vorticity vector field serves as the predictor and the pressure gradient (in the perpendicular plane) serves as the corrector. We describe an economical description of the vortex tube's cross-section: a 5-term truncated Fourier series is generally sufficient, and it compresses the representation of the flow by a factor of 4000 or more. We reconstruct the vortex tubes as generalized cylinders, providing a polygonal mesh suitable for display on a graphics workstation. We show how the reconstructed geometry of vortex tubes can be enhanced to help visualize helical motion in'a static image.
\end{abstract}

\footnotetext{
* This author was supported by the National Aeronautics and Space Administration under NASA contract No.NAS1-19480 while the author was in residence at the Institute for Computer Applications in Science and Engineering (ICASE), NASA
Langley Research Center, Hampton, VA 23681 .

** This author was supported by the Theoretical Flow Physics Branch at NASA Langley Research Center under contract
NAS1-19299.
} 



\section{Introduction}

Vortices are considered the most important structures that control the dynamics of flow fields. Large-scale vortices are responsible for hurricanes and tornadoes. Medium-scale vortices affect the handling characteristics of an airplane. Small-scale vortices are the fundamental building blocks of the structure of turbulent flow. One would like, therefore, to visualize a flow by locating all of its vortices and displaying them.

This paper presents a novel predictor-corrector technique for locating vortex structures in three-dimensional flow data. The technique is effective at locating vortices even in turbulent flow data. As an additional benefit, the technique provides a terse, one-dimensional representation of vortex tubes which offers significant compression of the flow data. Such compression is important if one wishes to visualize unsteady (i.e., time-varying) flows interactively.

Section 2 presents a survey of the efforts by various other researchers to define mathematical characteristics satisfied by vortices. Section 3 presents our predictor-corrector scheme for identifying vortices and discusses some of the programming considerations that are necessary to make the scheme efficient. Section 4 describes how we calculate the cross-sections of the vortex tube and how we represent them. In section 5 we show how the vortex skeletons, together with an efficient representation of the cross-sections, offer a substantial amount of data compression to represent features of a flow. We then describe the process of reconstructing the vortex tubes from the compressed format and show an enhanced reconstruction that helps visualize the motion of the fluid along the vortex tube.

\section{Survey of Identification Schemes}

The term "vortex" connotes a similar concept in the minds of most fluid dynamicists: a helical pattern of flow in a localized region. There are mathematical definitions for "vorticity" and "helicity," but vortical flow is not completely characterized by them. A precise definition is difficult to obtain - a fact supported by the variety of efforts outlined below.

\section{Spiral Moving With the Core}

Robinson [1] suggests the following working definition for a vortex.

A vortex exists when instantaneous streamlines mapped onto a plane normal to the vortex core exhibit a roughly circular or spiral pattem, when viewed from a reference frame moving with the center of the vortex core. 
Robinson [2] and Robinson, Kline, and Spalart [3] use the above rigorous definition to confirm that a particular structure is, in fact, a vortex. Unfortunately, this definition requires a knowledge of the vortex core before one can determine whether something is a vortex.

\section{Low Pressure}

Robinson and his colleagues find that elongated low-pressure regions in incompressible turbulent flows almost always indicate vortex cores. Isosurfaces of low pressure are usually effective at capturing the shape of an individual vortex (Figure la). Pressure surfaces become indistinct where vortices merge, however, and a high-quality image can easily require thousands of triangles to create the surface.

\section{Vorticity Lines}

Vorticity is a vector quantity that is proportional to the angular velocity of a fluid particle. It is defined as

$$
\omega=\nabla \times \mathbf{u}
$$

where $\mathbf{u}$ is the velocity at a given point. Vorticity lines are integral curves of vorticity (Figure 4). Moin and Kim [4] [5] use vorticity lines to visualize vortical structures in turbulent channel flow. The resulting curves are extremely sensitive to the choice of initial location $\mathbf{x}_{0}$ for the integration. As Moin and Kim point out [4],

If we choose $x_{0}$ arbitrarily, the resulting vortex line is likely to wander over the whole flow field like a badly tangled fishing line, and it would be very difficult to identify the organized structures (if any) through which the line may have passed.

They illustrate the potential tangle in Figure 2 of [5]. To avoid such a confusing jumble, they carefully select the initial points. However, Robinson [2] shows that even experienced researchers can be surprisingly misled by ordinary vorticity lines.

\section{Cylinder With Maximum Vorticity}

Villasenor and Vincent [6] present an algorithm for locating elongated vortices in threedimensional time-dependent flow fields. They start from a seed point and compute the average length of all vorticity vectors contained in a small-radius cylinder. They repeat this step for a large number of cylinders that emanate from the seed point. The cylinder with the maximum average becomes a segment of the vortex tube. They use only the magnitudes (not the directions) of vorticity; as a consequence the algorithm can inadvertently capture structures that are not vortices. 

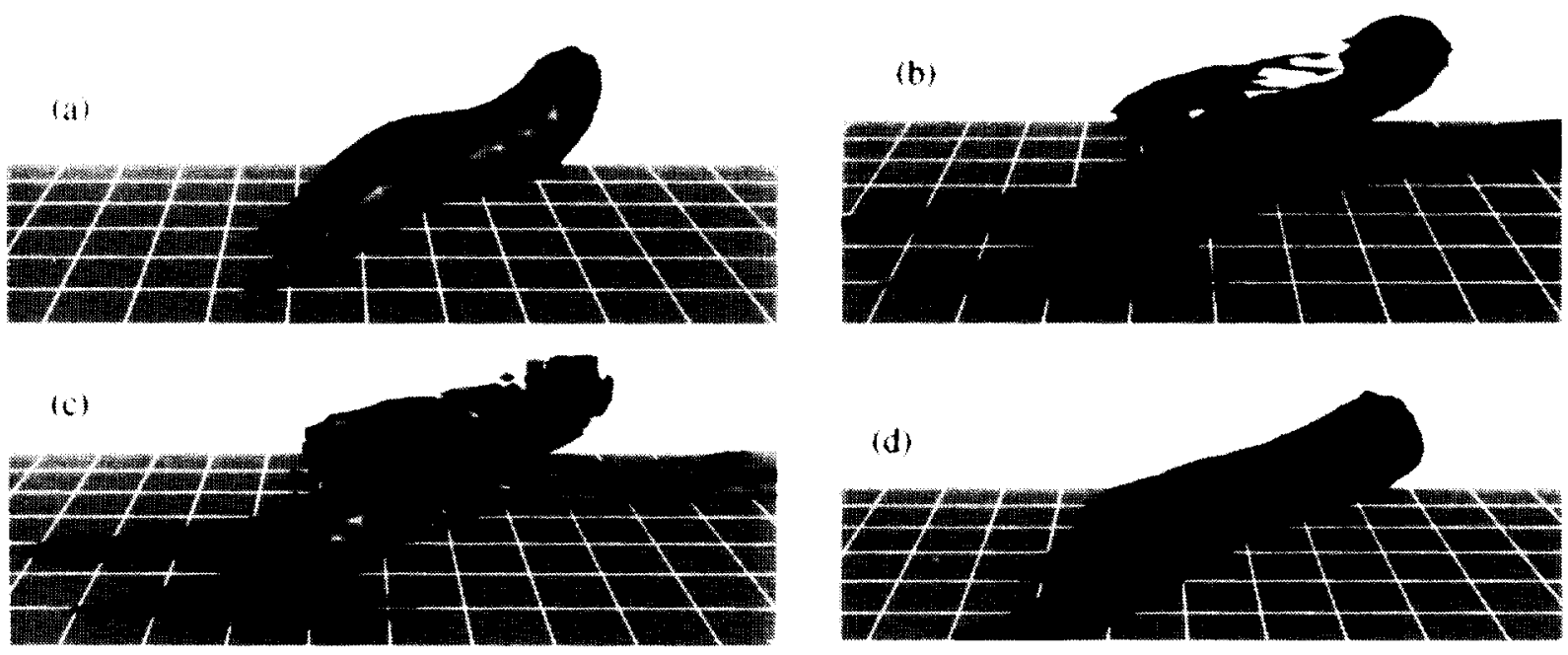

(c)

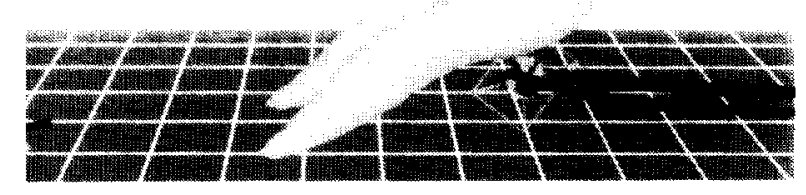

Figure 1. Different schemes used to identify a vortex. From upper left: (a) isosurface of constant pressure; (b) isosurfaces of constant vorticity; (c) isosurfaces of complex eigenvalues of the velocity-gradient matrix; (d) isosurface of constant helicity; (e) spiral-saddles (dark lines) compared with isopressure vortex tube (pale surface). Each image visualizes the same flow.

\section{Vorticity and Vortex Stretching}

Zabusky et al. [7] use vorticity $|\omega|$ and vortex stretching $|\omega \cdot \nabla \mathbf{u}| /|\omega|$ in an effort to understand the dynamics of a vortex reconnection process. They fit ellipsoids to the regions of high vorticity. Vector field lines of vorticity and of vortex stretching emanate from the ellipsoids. In flows with solid boundaries or a mean straining field, the regions with large vorticity magnitudes do not necessarily correspond to vortices (Figure 1b); hence, the ellipsoids do not always provide useful information.

\section{Velocity Gradient Tensor}

Chong, Perry, and Cantwell [8] define a vortex core as a region where the velocity-gradient tensor has complex eigenvalues. In such a region, the rotation tensor dominates over the rateof-strain tensor. Soria and Cantwell [9] use this approach to study vortical structures in freeshear flows. At points of large vorticity, the eigenvalues of the velocity-gradient matrix are determined: a complex eigenvalue suggests the presence of a vortex. 
This method correctly identifies the large vortical structures in the flow. However, the method also captures many smaller structures without providing a way to link the smaller vortical volumes with the larger coherent vortices of which they might be a part (Figure 1c).

\section{Curvature and Helicity}

Yates and Chapman [10] carefully explore two definitions of vortex cores. Unfortunately, the analyses and conclusions for both definitions are appropriate only for steady flows. By one definition, the vortex core is the line defined by the local maxima of normalized helicity. Figure $1 \mathrm{~d}$ shows an isosurface of constant helicity. Notice that the surface fails to capture the "head" on the upper-right side of the hairpin vortex. This shows that the local maxima fail to follow the core.

In the other definition, a vortex core is an integral curve that has minimum curvature. If there is a critical point on a vortex core, then that point must be a spiral-saddle. The eigenvector belonging to the only real eigenvalue of the spiral-saddle corresponds, locally, to an integral curve entering or leaving the critical point. By integrating this curve, the entire vortex core may be visualized [11]. Figure 1e, however, shows that these curves can miss the vortex completely. (The red spot is a critical point; the integral curves are colored blue).

\section{User-guided Search}

Bernard, Thomas, and Handler [12] use a semi-automated procedure to identify quasi-streamwise vortices. Their method finds local centers of rotation in user-specified regions in planes perpendicular to the streamwise direction of a turbulent channel flow. Experienced users can correctly find the critical vortices responsible for the maintenance of the Reynolds stress. Their method captures the vortices that are aligned with the streamwise direction, but in freeshear layers and transitional boundary layers, the significant spanwise vortices go undetected. Because it depends heavily on user intervention, the process is tedious and is dependent upon the individual skill of the user.

\section{The Predictor-corrector Method}

The methods listed above all experience success in finding vortices under certain flow conditions. But all of them have problems capturing vortices in unsteady shear flow, which is of interest to us because of its importance in understanding the transition from laminar to turbulent flow. We were led, therefore, to develop another technique which could tolerate the complexity of such a transitional flow. 
Our predictor-corrector method produces an ordered set of points that approximates a vortex skeleton. Associated with each point are quantities that describe the local characteristics of the vortex. These quantities may include the vorticity, the pressure, the shape of the cross-section, or other quantities of interest. This method produces lines that are similar to vorticity lines, but with an important difference. Whereas vorticity is a mathematical function of the instantaneous velocity field, a vortex is a physical structure with coherence over a region of space. In contrast to vorticity lines (which may wander away from the vortex cores), our method is self-correcting: line trajectories that diverge from the vortex core reconverge to the center.

In this section we discuss the procedure used to find an initial seed point on the vortex skeleton. We then explain the predictor-corrector method used for growing the vortex skeleton from the seed point. Finally, we address how to terminate the vortex skeleton.

\subsection{Finding a Seed Point}

Vorticity lines begin and end only at domain boundaries, but actual vortices have no such restriction. Therefore we must examine the entire flow volume in order to find seed points from which to initiate vortex skeletons. We consider low pressure and a large magnitude of vorticity to indicate that a vortex is present. Low pressure in a vortex core provides a pressure gradient that offsets the centripetal acceleration of a particle rotating about the core. Large vorticity indicates that such rotation is probably present.

In our implementation, the flow field (a three-dimensional rectilinear grid) is scanned in planes perpendicular to the streamwise direction. The scanning direction affects the order in which vortices are located, but not the overall features of the vortices. In each plane, the values of the pressure and the vorticity magnitude are checked against threshold values of these quantities. (Threshold values can be chosen a priori, or they can be a predetermined fraction of the extrema.) A seed point is a grid point that satisfies the two threshold values.

We next refine the position of the seed point so that it is not constrained to lie on the grid. The seed point moves in the plane perpendicular to the vorticity vector until it reaches the location of the local pressure minimum. From this seed point we develop the vortex skeleton in two parts: forward and backward.

\subsection{Growing the Skeleton}

Once a seed point has been selected, the skeleton of the vortex core can be grown from the seed. This is where we apply the two-stage predictor-corrector method. With this technique, the next position of the vortex skeleton is predicted by integrating along the vorticity vector. This candidate location is corrected by adjusting its position to lie at the pressure minimum in the plane that is perpendicular to the (original) vorticity vector. The rationale is that rotation about the vortic- 

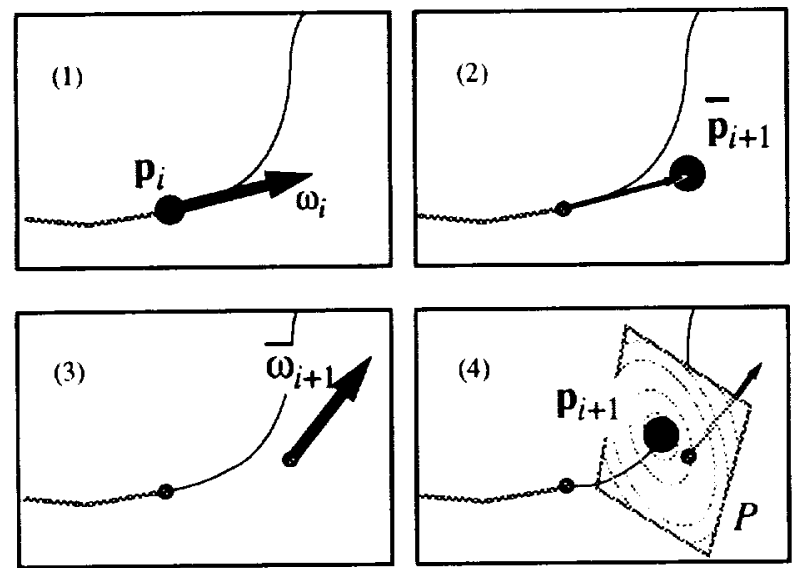

Figure 2. Schematic of predictor-corrector algorithm. foreach remaining seed point $\mathbf{p}_{0}$

if $p_{0}$ is not in any previous vortex

while the vortex skeleton continues

(1) determine $\omega_{i}$ at $\mathbf{p}_{i}$

(2) integrate $\omega_{i}$ to find $\bar{p}_{i+1}$

(3) determine vorticity $\bar{\omega}_{i+1}$ at $\overline{\mathbf{p}}_{i+1}$

(4) $\mathbf{p}_{i+1}$ is the point of minimum pressure in the plane $P \perp \bar{\omega}_{i+1}$

(5) $i \leftarrow i+1$

Predictor-corrector pseudocode.

ity vector is supported by low pressure at its center: the vortex tube's cross-section has its lowest pressure at the center of the tube. Where vortices merge this rule may be violated, causing the corrector step to lead the skeleton away from the vortex tube. To ensure that the minimum is actually part of the vortex under consideration, we limit the allowable angle between the vorticity vectors at the predicted and the corrected point.

Integral curves of vorticity or of the pressure gradient are both unreliable at capturing vortex skeletons. Remarkably, the combination of the two provides a robust method of following the vortex core. The continuous modification of the skeleton point lessens the sensitivity to both the initial conditions and the integration details.

The predictor-corrector algorithm is illustrated in the schematic diagrams of Figure 2 . The details for continuing the calculation from one point to the next are indicated by the numbered items in the pseudocode. Steps 1-2 represent the predictor stage of the algorithm. The corrector stage is summarized by steps 3-4.

The effectiveness of the predictor-corrector scheme is illustrated in Figure 3, in which data from the direct numerical simulations of Singer and Joslin [13] are analyzed. The transparent vortex tube (a portion of a hairpin vortex) is constructed with data from the full predictor-corrector method. Its core is indicated by the darker skeleton. The

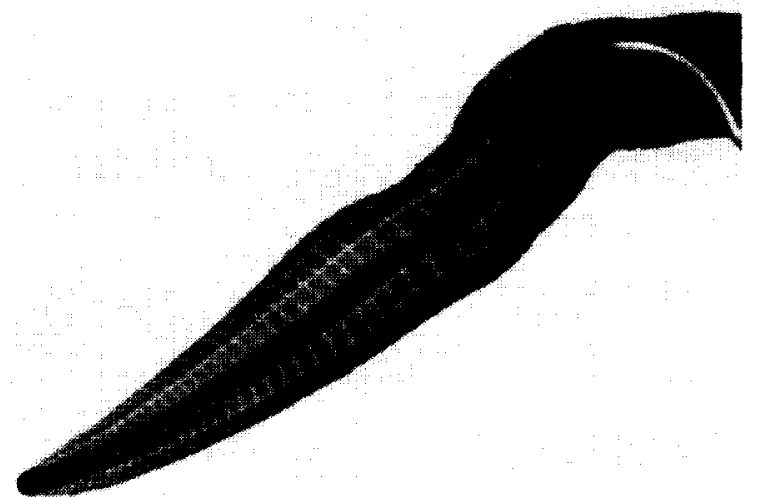

Figure 3. Vorticity line (light) compared to predictor-corrector line (dark). Note that the vorticity line exits from the vortex tube while the predictor-corrector skeleton line follows the core. 


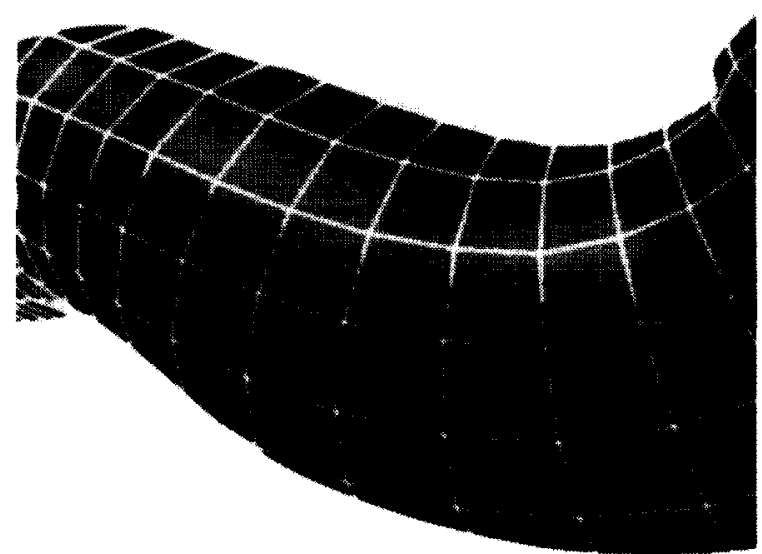

Figure 4. Multiple realizations of the same vortex tube from different seed points. Each seed point generates a slightly different skeleton line, although all the skeletons remain close to the vortex core.

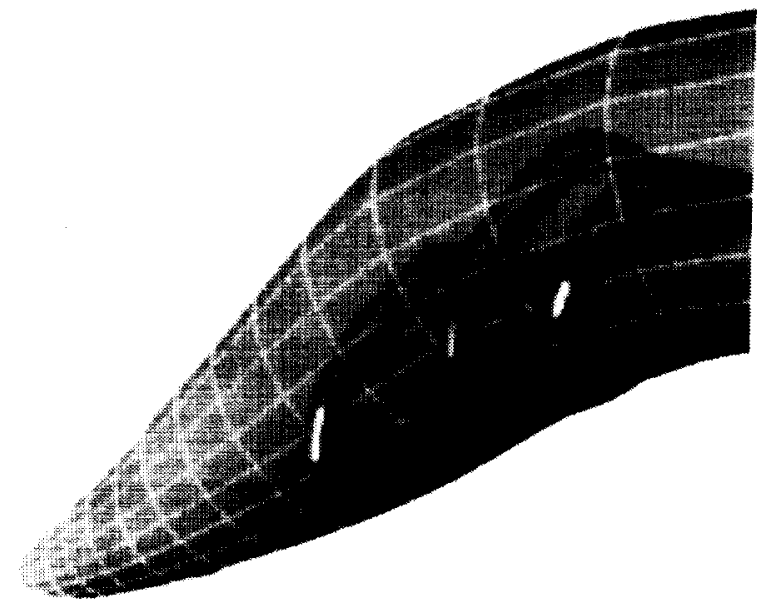

Figure 5. Feeders merge with a large-scale hairpin vortex. Three points that satisfy the threshold criteria lie on the edge of vortex tube. Their trajectories curve inward toward the core and then follow the main skeleton line.

lighter skeleton follows the uncorrected integral curve of the vorticity. It is obtained by disabling the corrector phase of the scheme. The vorticity line deviates from the core, exits the vortex tube entirely, and wanders within the flow field.

\subsection{Terminating the Vortex Skeleton}

Vorticity lines extend until they intersect a domain boundary, but real vortices typically begin and end inside the domain. Therefore, the algorithm must always be prepared to terminate a given vortex skeleton. A simple and successful condition for termination occurs when the vortex cross-section (discussed in section 4) has zero area. As Figures 3 and 7 show, the reconstructed vortex tubes taper down to their endpoints, where the cross-section vanishes.

\subsection{Implementation Details}

Although the general behavior of the predictor-corrector algorithm is reliable and robust, optimal performance of the technique requires careful attention to implementation details. This section addresses issues that are important to the successful use of this method. It is by no means exhaustive; additional details are provided by Singer and Banks [15].

\section{Eliminating Redundant Seeds and Skeletons}

Sampling every grid point produces an overabundance of seed points, and hence a multitude of nearly-coincident vortex skeletons (Figure 4). Each of these skeletons lies at the core of the 
very same vortex tube; one representative skeleton suffices. The redundancies are eliminated when points inside a tube are excluded from the pool of future seed points.

\section{Eliminating Spurious Feeders}

A seed near the surface of the vortex tube can produce a "feeder" vortex skeleton that spirals toward the vortex center. Examples of these feeders are illustrated in Figure 5. We eliminate feeders by taking advantage of the asymmetry of the predictor-corrector method. A feeder skeleton, begun on the surface of the tube, grows toward the core; a skeleton growing along the core does not exit through the tube. To validate a candidate seed $\mathbf{p}_{0}$, we integrate forward $n$ steps to the point $\mathbf{p}_{n}$ and then backward again by $n$ steps to determine a "true" seed point within the vortex.

\section{Numerical Considerations for Interpolation}

Neither the predictor nor the corrector step is likely to land precisely on a grid point; hence, we must interpolate the pressure and vorticity at arbitrary locations in the flow field. To reduce any bias from the interpolation, a four-point Lagrange interpolation is used in each of the three coordinate directions. The interpolation scheme works quite well, although it is the most expensive step in our implementation.

\section{Corrector Step}

The pressure-minimum correction scheme uses the method of steepest descent to find the local pressure minimum in the plane perpendicular to the vorticity vector. The smallest gridcell dimension is used as a local length scale to march along the gradient direction. The corrector stage can be iterated in order to converge to the skeleton, but that convergence is not guaranteed. We therefore limit the angle that the vorticity can change during the corrector phase.

\section{Finding the Cross-section}

Since it is unclear how to precisely define what points lie in a vortex, it is also unclear how to determine the exact shape of a vortex tube's cross-section. Determining an appropriate measure of the vortex cross-section has been one of the more difficult practical aspects of this work.

A point on the vortex skeleton serves as a convenient center for a polar coordinate system in the plane perpendicular to the skeleton line. We have chosen therefore to characterize the cross-section by a radius function. Note that this scheme correctly captures only star-shaped 
cross-sections. Cross-sections with more elaborate shapes are thereby truncated to star shapes (with discontinuities in the radius function). In practice this choice does not seem to be very restrictive, as section 4.2 indicates.

In examining the cross-section plane there are two important questions to address. First, what determines whether a point in the plane belongs to the vortex tube? Second, how should the shape of the tube's cross-section be represented? This section summarizes the strategies that we found to be successful.

\subsection{Criteria for Determining Membership}

For isolated vortices, a threshold of pressure provides an effective criterion to determine whether a point belongs to a vortex. When two or more vortices interact, their low-pressure regions merge and distort the radius estimate of any single vortex. This difficulty is resolved if the angle between the vorticity vector on the skeleton line and the vorticity vector at any radial position is restricted. Any angle greater than 90 degrees indicates that the fluid at the radial position is rotating in the direction opposite to that in the core. We have found that the 90 -degree restriction works well in combination with a low-pressure criterion for the vortex edge.

For the actual computation of the radial distance, the pressure and the vorticity are sampled along radial lines, emanating from the skeleton, lying in the perpendicular plane. We step along each radial line until a point is reached that violates the vorticity or the pressurethreshold criterion.

\subsection{Representation of the Cross-section}

If the radius of the cross-section were sampled at 1-degree increments, then 360 radial distances (and a reference vector to define the 0-degree direction) must be associated with each skeleton point. That is a great deal of data to save for each point of a time-varying set of vortex skeletons. We have found that average radius is sufficient to describe the cross-section of an isolated vortex tube.

When vortices begin to interact, the cross-section is non-circular and so the average radius does not provide a good description of it. However, a truncated Fourier series of the radial locations provides a convenient compromise between the average radius and a full set of all radial locations. The series is easy to compute, easy to interpret, and allows a large range of cross-sectional shapes. In our work, we keep the constant term, the first and second sine and cosine coefficients, and a reference vector. Most of the cases that we have checked have a 


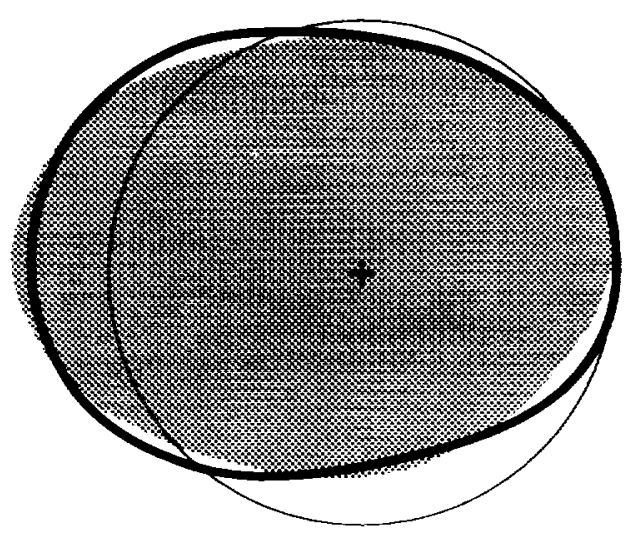

Figure 6. Cross-section of a vortex tube. The finelysampled radius function is shaded grey. The thin line is an approximating circle. The thick line is a truncated Fourier representation.

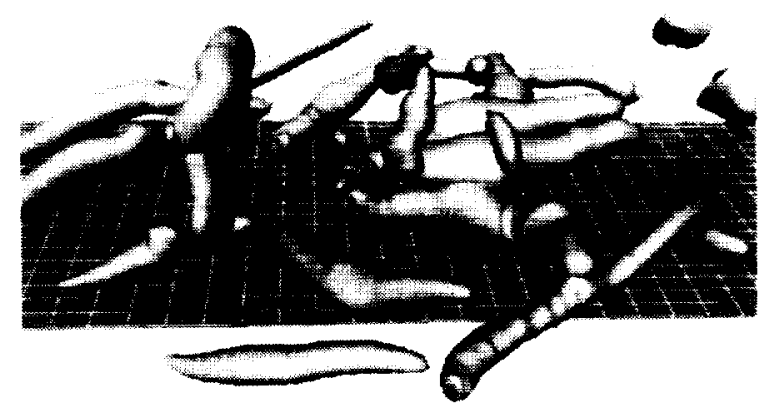

Figure 7. Interacting vortices (from a numerical simulation) within a complex flow are identified with the predictor-corrector algorithm. The direction of flow is to the right.

factor-of-10 drop in the magnitude of the first and second coefficients, indicating that the neglected terms are not significant. That observation also validates our assumption that the cross-section is well-represented by a continuous polar function.

Figure 6 illustrates a single cross section of a vortex educed from direct numerical simulation data. The shaded region is the interior of the vortex tube, sampled at 1-degree intervals. The thin line is a circle, centered at the skeleton, using the averaged radius of the vortex tube. The thick line is the truncated Fourier series representation of the vortex cross-section, providing a better approximation than the circle.

\section{Data Compression and Reconstruction}

Our particular interest is to visualize the transition to turbulence in a shear flow. We have performed a lengthy simulation using Cray computers over the course of two calendar years. The numerical grid grows with the size of the evolving flow structures, but a grid size of $461 \times 161 \times 275$ in streamwise, wall-normal, and spanwise directions is representative. Each grid point holds several numerical quantities, including pressure and vorticity. Thus the storage exceeds $650 \mathrm{MB}$ (megabytes) of data per time step.

By using vortex skeletons we are able to compress the data significantly and then reconstruct the vortex tubes locally on a workstation. 


\subsection{Compression}

An animation of the vortices, based on the original computational volumetric data, would consume over $650 \mathrm{~GB}$ of data for 1000 time steps. At present this is a prohibitive requirement of an interactive 3D animation on a workstation. Storing all the individual polygons offers some compression, but not enough to bring an animation within reach.

In general, a vortex skeleton is adequately represented by 30 to 200 samples. The complex scene in Figure 7 is represented by about 2000 skeleton points, each endowed with 72 bytes of data (representing position, tangent, normal, binoimal, cross-section, and velocity magnitude). Thus a reduction from $648 \mathrm{MB}$ to $144 \mathrm{~KB}$ is achieved, representing more than a 4000 )fold factor of compression. This amount of compression offers the promise of workstationbased interactive animations, even for a 1000 -frame simulation.

\subsection{Reconstruction}

The significant data-compression that vortex skeletons provide does not come without cost. There is still the matter of reconstructing polygonal tubes from the skeletons. If the tubes have circular cross-sections, they are generalized cylinders. Bloomenthal gives a clear exposition of how to reconstruct a generalized cylinder from a curve through its center [14]. The coordinate system of the cross-section rotates from one skeleton point to the next. The key issue is how to keep the rate of rotation (about the skeleton's tangent vector) small. Excessive twist is visible in the polygons that comprise the tube: they become long and thin and their interiors approach the center of the tube.

In our implementation, we project the coordinate bases from one cross-section onto the the next cross-section. This produces a new coordinate system that has not twisted very much. This normal vector might be different from the reference vector (which indicates the 0-degree direction) for the Fourier representation of the cross-section. To reconstruct the cross-section, we phase-shift the angle in the Fourier series by the angular difference between the normal and the reference vector. In general, 30 to 80 samples suffice to reconstruct a cross-section of good quality.

Sometimes there is good reason for a "reconstruction" that is not faithful to the original shape of the vortex tube. A static image does not convey the spiraling motion along the surface of the vortex tube. We experimented with different methods of visualizing the velocities on the tube itself. One helpful technique is to create a texture on the surface, drawing curves to indicate the helical flow. This visualization is enhanced dramatically when the curves are dis- 


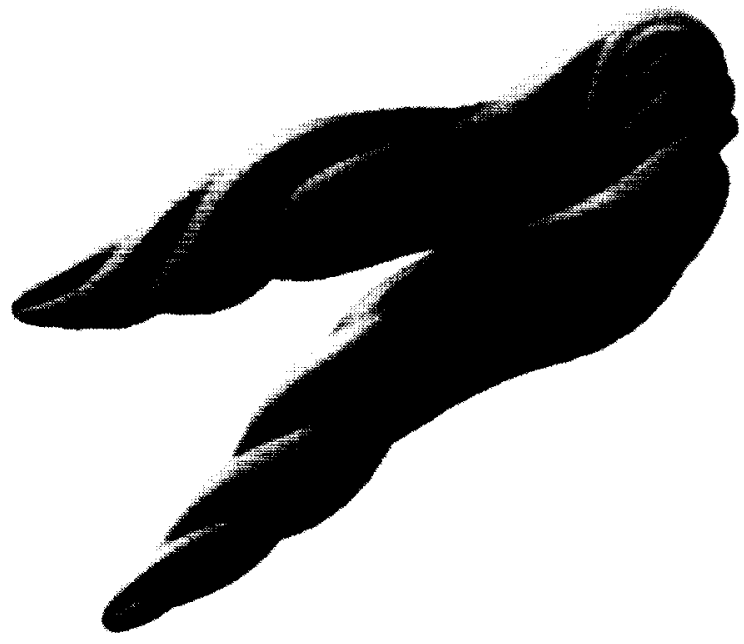

Figure 8. Enhanced reconstruction of a hairpin vortex tube. The grooves follow integral curves of velocity, constrained to follow the surface of the tube. Green indicates large angular velocity; red indicates small angular velocity. placed inward to produce grooves. Figure 9 demonstrates this technique on a single hairpin vortex. The grooves follow integral curves of the surface-constrained velocity vectors. In an informal survey of about a dozen colleagues, we found that none could estimate the amount of helical motion in a faithful reconstruction (as in Figures 4 and 8 ) of a vortex tube. On the other hand, the same subjects instantly identified the direction and amount of rotation in the enhanced image of Figure 8.

There are two important issues in reconstruction that we have not yet addressed in our implementation; both relate to the rep-

resentation and compression of the vortices as well. First, we would like to minimize the number of samples carried by a vortex skeleton. Where the vortex skeleton has high curvature or where the cross-section changes shape quickly, many samples are required to permit an accurate reconstruction. But most vortex tubes have long, straight portions with nearly-circular cross-sections of nearly-constant radius. This characteristic should permit us to represent the vortex tube with many fewer samples along its skeleton.

The second issue concerns interpolation. In reviewing the development of a vortical flow, a scientist may be especially interested in narrowing the interval of animation to only a few of the original time steps. It would be helpful to generate in-between frames from the given data. We could interpolate the original volumetric grids to extract interpolated vortex skeletons, but that would require a great deal of data communication. Interpolating between the skeletal representations, on the other hand, could be done in memory. Unfortunately, it is difficult to interpolate between irregular branching structures like the time-varying vortex skeletons. These two issues remain as future work.

\section{Conclusions}

The innovative use of a two-step predictor-corrector algorithm has been introduced to identify vortices in flow-field data. Unlike other approaches, our method is able to self-correct toward 
the vortex core. The principle of using a vector field to predict the location of the next point and a scalar field to correct this position distinguishes this method from others.

This paper discusses a number of novel approaches that we have developed to deal with matters such as eliminating redundant vortices, eliminating feeders, and representing the crosssection of a vortex tube. Sample extractions of vortices from various flow fields illustrate the different aspects of the technique.

The vortex skeletons are an economical way to represent flow data, offering a 4000-fold compression factor even in a complex flow. This offers the possibility of storing a multi-frame flow animation in a workstation's memory. The vortex tubes can be enhanced during reconstruction in order to help visualize the dynamics of vortical flow.

\section{Acknowledgments}

The images in Figure 1 were rendered on a Silicon Graphics Indigo workstation using the FAST visualization system. The images in Figures 3, 4, 5, and 7 were rendered on a Silicon Graphics Indigo2 using the Explorer visualization system. The image in Figure 8 was rendered on an Intel Paragon using PGL (Parallel Graphics Library), which was developed at ICASE by Tom Crockett and Toby Orloff.

We thank Gordon Erlebacher for participating in the early discussions of vortex identification schemes and for making helpful suggestions.

\section{References}

[1] S. K. Robinson, "Coherent motions in the turbulent boundary layer," Annu. Rev. Fluid Mech. 23, 601 (1991).

[2] S. K. Robinson, "A review of vortex structures and associated coherent motions in turbulent boundary layers," in Proceedings of Second IUTAM Symposium on Structure of Turbulence and Drag Reduction, Federal Institute of Technology, Zurich, Switzerland, July 25-28 (1989).

[3] S. K. Robinson, S. J. Kline, and P. R. Spalart, "A review of quasi-coherent structures in a numerically simulated boundary layer,“ NASA TM-102191 (1989).

[4] P. Moin and J. Kim, "The structure of the vorticity field in turbulent channel flow. Part 1. Analysis of instantaneous fields and statistical correlations,“ J. Fluid Mech. 155, 441 (1985). 
15] J. Kim, and P. Moin, "The structure of the vorticity field in turbulent channel flow. Part 2. Study of ensemble-averaged fields," J. Fluid Mech. 162, 339 (1986).

[6] J. Villasenor and A. Vincent, "An algorithm for space recognition and time tracking of vorticity tubes in turbulence," CVGIP: Image Understanding 55:1, 27 (1992).

171 N. J. Zabusky, O. N. Boratav, R. B. Pelz, M. Gao, D. Silver, and S. P. Cooper, "Emergence of coherent patterns of vortex stretching during reconnection: A scattering paradigm," Phys. Rev. Let. 67:18, 2469 (1991).

[8] M. S. Chong, A. E. Perry, and B. J. Cantwell, "A general classification of three-dimensional flow fields," Phys. of Fluids A 2:5, 765 (1990).

191 J. Soria, and B. J. Cantwell, "Identification and classification of topological structures in free shear flows, " in it Proceedings of IUTAM Eddy Structure Identification in Free Turbulent Shear Flows (1992).

|10| L. A. Yates, and G. T. Chapman, "Streamlines, Vorticity Lines, and Vortices," AIAA Paper 91-0731 (1991).

111] A. Globus, C. Levit, and T. Lasinski, "A Tool for Visualizing the Topology of ThreeDimensional Vector Fields," Report RNR-91-017, NAS Applied Research Office, NASA Ames Research Center (1991).

[12] P. S. Bernard, J. M. Thomas, and R. A. Handler, "Vortex dynamics and the production of Reynolds stress,“ II. Fluid Mech. 253, 385 (1993).

[13] B. A. Singer and R. D. Joslin, "Metamorphosis of hairpin vortex into a young turbulent spot," submitted to Phys. Fluids A (1993).

[14] Jules Bloomenthal, "Calculation of Reference Frames Along a Space Curve," Graphics Gems I, (Andrew Glassner, ed.), Academic Press, INC. (1990).

$115]$ B. A. Singer and D. C. Banks, "A Predictor-Corrector Scheme for Vortex Identification," ICASE Report No. 94-11; NASA CR-194882 (1994). 





\begin{tabular}{|c|c|c|c|}
\hline \multicolumn{3}{|c|}{ REPORT DOCUMENTATION PAGE } & $\begin{array}{l}\text { Form Approved } \\
\text { OMB No 0704-0188 }\end{array}$ \\
\hline \multicolumn{4}{|c|}{ 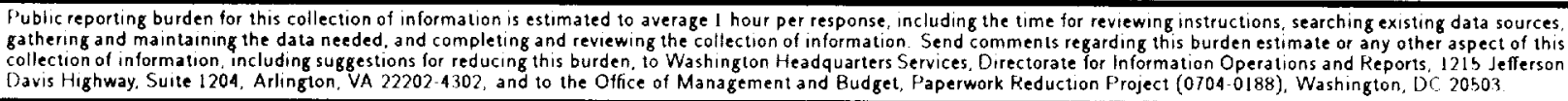 } \\
\hline 1. AGENCY USE ONLY(Leave biank) & $\begin{array}{l}\text { 2. REPORT DATE } \\
\text { April } 1994\end{array}$ & \multicolumn{2}{|c|}{$\begin{array}{l}\text { 3. REPORT TYPE AND DATES COVERED } \\
\text { Contractor Report }\end{array}$} \\
\hline \multicolumn{3}{|c|}{$\begin{array}{l}\text { 4. TITLE AND SUBTITLE } \\
\text { VORTEX TLBES IN TURBULENT FLOWS: ITENTIFICATIÓN, } \\
\text { REPRESENTATION, RECONSTRUCTION }\end{array}$} & \multirow[t]{2}{*}{$\begin{array}{l}\text { 5. FUNDING NUMBERS } \\
\text { ( NAS1-19480 } \\
\text { WU } 505-90-52-01\end{array}$} \\
\hline \multicolumn{3}{|l|}{$\begin{array}{l}\text { 6. AUTHOR(S) } \\
\text { David (:. Banks } \\
\text { Bart A. Singer }\end{array}$} & \\
\hline \multicolumn{3}{|c|}{$\begin{array}{l}\text { 7. PERFORMING ORGANIZATION NAME(S) AND ADORESS(ES) } \\
\text { Institute for Computer Applications in Science } \\
\text { and Engineering } \\
\text { Mail Stop } 132(\text {, NASA Langley Research Center } \\
\text { Hampton, VA } 23681-0001\end{array}$} & $\begin{array}{l}\text { 8. PERFORMING ORGANIZATION } \\
\text { REPORT NUMBER } \\
\text { ICASE REPOIT No, } 94-22\end{array}$ \\
\hline \multicolumn{3}{|c|}{$\begin{array}{l}\text { 9. SPONSORING/MONITORING AGENCY NAME(S) AND ADDRESS(ES) } \\
\text { National Aeronautics and Space Administration } \\
\text { Langley Research Center } \\
\text { Hampton, VA } 23681-0001\end{array}$} & $\begin{array}{l}\text { 10. SPONSORING/MONITORING } \\
\text { AGENCY REPORT NUMBER } \\
\text { NASA CR-194900 } \\
\text { ICASE RepOrT No. } 94-22\end{array}$ \\
\hline \multicolumn{4}{|c|}{$\begin{array}{l}\text { 11. SUPPLEMENTARY NOTES } \\
\text { Langley Technical Monitor: Michael F. Card } \\
\text { Final Report } \\
\text { Subnitted to the October Meeting of Visualization ' } 94\end{array}$} \\
\hline \multicolumn{2}{|c|}{$\begin{array}{l}\text { 12a. DISTRIBUTION/AVAILABILITY STATEMENT } \\
\text { Dinclassified Inlimited } \\
\text { Sinbject Category 60,34 }\end{array}$} & & 12b. DISTRIBUTION CODE \\
\hline \multicolumn{4}{|c|}{$\begin{array}{l}\text { 13. ABSTRACT (Maximum } 200 \text { words) } \\
\text { In many cases the structure of a fluid flow is well-characterized by its vortices, especially for the purpose of } \\
\text { visualization. In this paper we present a new algorithm for identifying vortices in complex flows. The algorithm } \\
\text { produces a skeleton line along the center of a vortex by using a two-step predictor-corrector scheme. The vorticity } \\
\text { vector field serves as the predictor and the pressuregradient (in the perpendicular plane) serves as the corrector. We } \\
\text { describe an economical description of the vortex tube's cross-section: a } 5 \text {-term truncated Fourier series is generally } \\
\text { sufficient, and it compresses the representation of the flow by a factor of } 4000 \text { or more. We reconstruct the vortex } \\
\text { tubes as generalized cylinders, providing a polygonal mesh suitable for display on a graphics workstation. We show } \\
\text { how the reconstructed geometry of vortex tubes can be enhanced to help visualize helical motion in a static image. }\end{array}$} \\
\hline \multirow[t]{2}{*}{$\begin{array}{l}\text { 14. SUBJECT TERMS } \\
\text { Vortex, visualization }\end{array}$} & & 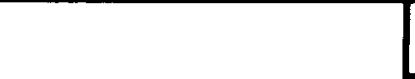 & $\begin{array}{l}\text { 15. NUMBER OF PAGES } \\
16\end{array}$ \\
\hline & & & $\begin{array}{c}\text { 16. PRICE CODE } \\
\text { A } 03\end{array}$ \\
\hline $\begin{array}{l}\text { 17. SECURITY CLASSIFICATION } \\
\text { OF REPORT } \\
\text { Unclassified }\end{array}$ & $\begin{array}{l}\text { 18. SECURITY CLASSIFICATION } \\
\text { OF THIS PAGE } \\
\text { Inclassified }\end{array}$ & $\begin{array}{l}\text { 19. SECURITY CLASSIFICATION } \\
\text { OF ABSTRACT }\end{array}$ & $\begin{array}{l}\text { 20. LIMITATION } \\
\text { OF ABSTRACT }\end{array}$ \\
\hline NSN 7540-01-280-5500 & & & $\begin{array}{l}\text { Standard Form } 298(\text { Rev. } 2-89) \\
\text { Prescribed by ANSI Std. } 239.18 \\
298.102\end{array}$ \\
\hline
\end{tabular}

